\title{
Zebrafish cardiac regeneration-looking beyond cardiomyocytes to a complex microenvironment
}

\author{
Rebecca Ryan $^{1} \cdot$ Bethany R. Moyse ${ }^{1} \cdot$ Rebecca J. Richardson $^{1}[0$
}

Accepted: 30 August 2020 / Published online: 14 September 2020

(c) The Author(s) 2020

\begin{abstract}
The study of heart repair post-myocardial infarction has historically focused on the importance of cardiomyocyte proliferation as the major factor limiting adult mammalian heart regeneration. However, there is mounting evidence that a narrow focus on this one cell type discounts the importance of a complex cascade of cell-cell communication involving a whole host of different cell types. A major difficulty in the study of heart regeneration is the rarity of this process in adult animals, meaning a mammalian template for how this can be achieved is lacking. Here, we review the adult zebrafish as an ideal and unique model in which to study the underlying mechanisms and cell types required to attain complete heart regeneration following cardiac injury. We provide an introduction to the role of the cardiac microenvironment in the complex regenerative process and discuss some of the key advances using this in vivo vertebrate model that have recently increased our understanding of the vital roles of multiple different cell types. Due to the sheer number of exciting studies describing new and unexpected roles for inflammatory cell populations in cardiac regeneration, this review will pay particular attention to these important microenvironment participants.
\end{abstract}

Keywords Zebrafish $\cdot$ Heart regeneration $\cdot$ Microenvironment $\cdot$ Immune cells $\cdot$ Cardiomyocytes

\section{Introduction}

Cardiovascular disease and myocardial infarction (MI) remain major global health burdens, and their relationship to the escalating obesity epidemic and an increasingly unhealthy lifestyle means their frequency is unlikely to decline in the coming years (Braunwald 2015). MI, often occurring as a consequence of coronary heart disease, can result in massive cardiomyocyte (CM) loss, perhaps up to 1 billion cells (Murry et al. 2006) and is often followed by fibrotic tissue and scar formation, severely limiting the functional capacity of the heart and leading to heart failure. The holy grail for cardiovascular therapeutics would be the ability to stimulate cardiac regeneration and replace the myocardium lost to cell death; however, this remains elusive and poorly understood, with regeneration in mammalian models

Rebecca J. Richardson

rebecca.richardson@bristol.ac.uk

1 C21a, Biomedical Sciences Building, Faculty of Life Sciences, School of Physiology, Pharmacology and Neuroscience, University of Bristol, University Walk, Bristol BS8 1TD, UK restricted to early developmental stages (reviewed by Murry et al. 2006; Cahill et al. 2017). Current treatment options for cardiovascular disease range from pharmaceutical to transplant; however, there is no escaping that each strategy has associated drawbacks (reviewed by Hashimoto et al. 2018).

The ability to somehow stimulate the production of new $\mathrm{CMs}$, for example via the use of stem cell or cell replacement therapies, has long been the ultimate goal (reviewed by Murry et al. 2006; Hashimoto et al. 2018). However, the limited success of CM-focused therapies has highlighted that these approaches are too narrow and overly focused on a single cell type. To develop an approach that could potentially stimulate endogenous regeneration or provide additional support to a CM replacement strategy, it will also be essential to harness a pro-regenerative microenvironment, widening the scope to encompass diverse non-myocytes and the extracellular milieu. To study this complexity, it seems logical to turn to a model system that offers both a natural regenerative ability and a complete in vivo system, criteria that are met (almost uniquely) by the zebrafish.

Surgical resection of the apex of the ventricle has been in use for almost twenty years to study the mechanisms involved in regenerating cardiac tissue in the adult zebrafish 
(Poss 2002). Additionally, genetic cell ablation models have been utilised to reduce $\mathrm{CM}$ number and trigger regeneration (Wang et al. 2011). More recently, the development of a cryoinjury model, whereby a liquid nitrogen-cooled probe is placed on the ventricle to induce localised cell death (Chablais et al. 2011; Schnabel et al. 2011; Gonzalez-Rosa et al. 2011), has provided a new aperture to view the resulting regeneration and is more representative of the cellular damage resulting from MI. Whichever injury model is chosen, studies into the complex injury response in this model are providing a wealth of information on the cellular and molecular mechanisms required to rebuild the heart after damage and are increasingly highlighting the need to widen the scope beyond CMs to the microenvironment surrounding these cells.

\section{Current therapeutic outlook}

Despite the relatively simple architecture of the adult mammalian heart, repair is complex, and regeneration has proven unattainable thus far. As regeneration is currently impossible, and heart transplant is limited by practical considerations (not least the lack of donors but also the associated surgical complexities) (Yacoub 2015), the therapeutic focus is treatment rather than cure, specifically in preventing the progression of ischaemic heart disease to heart failure (Sacks et al. 2014). Cardioprotective treatment options can improve blood supply (e.g. revascularisation by thrombolysis or bypass surgery) and pharmacological interventions can decelerate cardiac remodelling (e.g. ACE inhibitors and $\beta$-blockers), whereas more advanced stages of heart failure benefit from mechanical support therapies (such as left-ventricular assist devices or cardiac resynchronisation therapy) (Reviewed by Hashimoto et al. 2018). Despite this broad range of therapeutic interventions, ischaemic heart failure and its associated adverse cardiac remodelling remain major challenges for health services worldwide.

Cell-replacement therapies (using, for example, direct application of (often exogenous) stem cells or re-differentiated CMs derived from somatic cells via induced pluripotent stem cells (iPSCs)) aim to directly address the problem of insufficient $\mathrm{CM}$ proliferation, which is widely considered to be the limiting factor in heart regeneration. These therapies, which involve growing vast numbers of cells in vitro (potentially more than the 1 billion CMs that may be lost following MI) and injecting them directly into the injured heart, have shown promise in numerous pre-clinical settings. However, results for this relatively new strategy are often inconsistent and fail to achieve marked improvements in cardiac function (reviewed by Müller et al. 2018; Tehzeeb et al. 2019), and studies in animal models have revealed concerning and prevalent side effects including arrhythmias and tachycardia
(Shiba et al. 2016; Liu et al. 2018; and reviewed by Chen et al. 2020). These inconsistencies may be partly explained by a recent and important publication from Vagnozzi et al. (2020) which has found that direct chemical stimulation of the innate immune system produces an outcome comparable to treatment with cell therapies previously reported to be reparative in models of cardiac ischaemic injury (Vagnozzi et al. 2020). This comprehensive report has demonstrated that beneficial effects thought to result from stem cell therapies are chiefly mediated by an acute regional accumulation of specific macrophage populations and an inflammatory wound healing response triggered by the cell transplant, rather than direct proliferation of the transplanted cells. These findings are also cohesive with an emerging research trend to further delineate the role of the immune response in cardiac repair and regeneration, which will be the focus of this review.

\section{Cardiomyocyte proliferation}

In recent years, the scant proliferative capacity of CMs has been highlighted as the major limiting factor in the process of heart regeneration following cardiac injury/MI. In the mature mammalian heart, CM proliferation is poor, and progenitors are present in low numbers (reviewed by Cahill et al. 2017; Hashimoto et al. 2018), with the post-natal heart mainly increasing in size via cardiac hypertrophy (Alkass et al. 2015). The composition of the adult myocardium is noteworthy when considering its regenerative potential. Despite the fact that approximately $90 \%$ of the myocardial mass is composed of CMs, these cells only represent $~ 30 \%$ of the total number of cells, the rest being predominantly composed of endothelial cells ( 43\%), fibroblasts $(\sim 20 \%)$ and leukocytes ( 7\%) (Reiss et al. 1996; Pinto et al. 2016). About $1 \%$ are CM progenitor cells (or stem cells), and this, coupled with the fact that the majority of adult CMs are quiescent, is thought to account for the fact that the adult human heart has a very minimal regenerative capacity post-injury, with CMs estimated to have an annual renewal rate ranging from $1 \%$ in early adulthood ( 25 years) to as little as $0.45 \%$ in later life ( 75 years) (Murry et al. 2006). Considering the cellular damage post-MI can be in the region of a loss of $25 \%$ of cells (Murry et al. 2006), it is clear that normal turnover and replacement operates at a substantial deficit and is insufficient to restore a functional myocardium post-infarction (Bergmann et al. 2009; Senyo et al. 2013). However, if only $30 \%$ of the total number of cardiac cells are CMs, it seems restrictive to narrow our focus for regenerative capacity to this single cell type.

Despite the absence of robust regeneration in the adult mammalian heart, cardiac regeneration is observed, to varying degrees, in neonatal mammals. Murine and porcine 
hearts are capable of efficient regeneration, providing the injury occurs in the first 2 days post-natally, and the magnitude of the injury is not too great $(<15 \%)$ (Porrello et al. 2011; Bryant et al. 2015; Notari et al. 2018; Ye et al. 2018; Zhu et al. 2018). Use of an adult zebrafish cryoinjury model also supports this finding that even in highly regenerative systems the potential is not inexhaustible. Injury resulting in up to $20 \%$ ventricular cell death (by area) seems to be well tolerated and completely resolved by 60 -day post-injury (dpi) (Chablais et al. 2011; Bevan et al. 2020) yet increasing the injury area by $5 \%$ seems to push the regenerative capacity of the zebrafish heart beyond its limits, with scar resolution incomplete even at 130 dpi (Gonzalez-Rosa et al. 2011). Additionally, a recent report demonstrates that repeated cryoinjuries limit the regenerative ability of adult zebrafish (Bise et al. 2020). Interestingly, CM proliferation is activated after each injury (although this becomes less efficient over time), but collagen deposition is exacerbated by each injury and scar removal gradually fails, further suggesting that regenerative capacity has limits (Bise et al. 2020).

Zebrafish injury models have also been instrumental in clarifying the source of new heart muscle cells in a regenerative context. Several studies have exploited lineage-tracing methods to show that existing mature $\mathrm{CMs}$ are the source of new cardiac muscle and stem/progenitor cells have no significant involvement in this process (Jopling et al. 2010 and reviewed by Kikuchi 2015). Morphologically, the dividing zebrafish CMs change their contractile state in a manner similar to structural alterations observed to facilitate proliferation in murine cells, such as disassembly of sarcomeres, which may indicate conservation of the underlying processes required for CM proliferation (Ahuja et al. 2004; Jopling et al. 2010; and reviewed by Kikuchi 2015).

There is some CM proliferation in adult mammals and this likely arises from existing CMs (as in zebrafish); however, this occurs at a much lower frequency and the signature of the CMs retaining this proliferative ability has not yet been fully determined (Bergmann et al. 2009; Jopling et al. 2010; Senyo et al. 2013; Kikuchi 2015). Evidence has emerged in recent years to suggest that ploidy is an important factor. The majority of zebrafish CMs are mononucleated and diploid; however, these properties are lost in mammalian cells which become binucleated or polyploid soon after birth (Soonpaa et al. 1996; Kikuchi et al. 2010; Mollova et al. 2013; Ye et al. 2016). This hypothetical relationship has been further substantiated as the prevalence of mononuclear diploid CMs has been shown to correlate with functional recovery and CM proliferation after coronary artery ligation in mice and CM ploidy can generally predict regenerative potential across vertebrate species (Patterson et al. 2017; Hirose et al. 2019). Gonzalez-Rosa et al. have recently shown direct evidence of the importance of ploidy, as experimental polyploidisation of zebrafish CMs is sufficient to inhibit proliferative potential in this highly regenerative model, and a substantial proportion of diploid CMs (approximately 75\%) is required to support regeneration, leading the authors to propose stimulation of the rare diploid cells in the human heart as a method to boost heart regeneration (González-Rosa et al. 2018).

The importance of CM replacement for complete regeneration to occur is clear, but the value of the interplay between these cells and their microenvironment is just beginning to be elucidated. The study of many different in vivo models of cardiovascular disease and injury is helping researchers look beyond CMs to the myriad of cells and processes that co-activate, co-ordinate and co-regulate regeneration of the heart. These processes include signalling mechanisms for cell recruitment (reviewed by Sanz-Morejón and Mercader 2020), phagocytic immune cell removal of debris (de Preux Charles et al. 2016; Lai et al. 2017; Bevan et al. 2020), fibroblasts laying down ECM (Simões et al. 2020) and MMP breakdown of ECM to permit the infiltration of new vasculature (Bellayr et al. 2009; Marín-Juez et al. 2016; Xu et al. 2019), which ultimately provide a supportive architecture for the invasion of new CMs. Further study of this complex interplay will be key to our understanding of the regenerative process as a whole.

\section{Zebrafish as a model for repair \& regeneration}

Numerous advantages of the zebrafish, such as their high fecundity, external fertilisation and the transparency of developing larvae, have been fundamental in establishing them as a valuable model system for vertebrate developmental biology (reviewed by Dooley 2000). Further to this, their genetic tractability has proven an adaptable asset during an extraordinary transitional period in genome editing, from early labour-intensive reverse genetics approaches like ENU-screening through to the now ubiquitous CRISPR-cas9 system (Koster and Sassen 2015; Sertori et al. 2016). As a non-mammalian model, there are genetic discrepancies, with the poor conservation of some alleles and duplication of approximately $20 \%$ of genes (Postlethwait 2000) sometimes making direct comparisons difficult. However, $70 \%$ of protein-coding human genes are related to genes found in zebrafish, and $84 \%$ of disease-related genes have a zebrafish equivalent (Howe et al. 2013) meaning they have become an important model of human disease (reviewed by Lieschke and Currie 2007). Antibody availability has historically been poor (though in recent years the prevalence of custom synthesis services and the popularity of the zebrafish as a model system has led to some improvements on this score), yet amenability to genome-editing has also facilitated the generation of numerous transgenic lines which are both a 
powerful research tool in their own right and partially compensate for the lack of commercially available antibodies.

In addition to these well-known characteristics, the key attribute for zebrafish in this research context is their nearunique ability to completely regenerate the adult heart postinjury, thus providing a cellular and molecular map for the processes from repair to regeneration (Poss 2002; Chablais et al. 2011; Schnabel et al. 2011; Gonzalez-Rosa et al. 2011; Bevan et al. 2020). As described above, three main models of cardiac injury have been described in adult zebrafish, (1) cardiac resection (Poss 2002), (2) cryoinjury (Chablais et al. 2011; González-Rosa et al. 2011; Schnabel et al. 2011) and (3) genetic ablation of CMs (Wang et al. 2011). The resection model, which involves the surgical removal of the ventricular apex, was used in the first landmark study describing the regenerative ability of the adult zebrafish heart (Poss 2002). First published in 2011, the cryoinjury model arguably provides the most representative model for the ischaemia-induced cell death associated with infarction, resulting in extensive scar formation (Chablais et al. 2011; González-Rosa et al. 2011; Schnabel et al. 2011; Bevan et al. 2020). Although these initial cryoinjury studies report some discrepancies in the "completeness" of the regeneration process, this is likely due to differences in the extent of the injury inflicted (20\% ventricular area vs $25 \%$, Chablais et al. 2011; González-Rosa et al. 2011, respectively), and overall, these studies largely correlate on the major events post-injury. Around the same time as the description of the cryoinjury model, it was shown that adult zebrafish could also recover from the loss of up to $60 \%$ of their CMs, ablated using a genetic Cre/LoxP-driven cytotoxic diptheria toxin $\mathrm{A}$ expression system (Wang et al. 2011). Although this system further highlights the remarkable regenerative capacity of the zebrafish and allows a less invasive method to study CM replacement, it is arguably the least representative of human cardiac damage as it is not localised, resulting in the random loss of CMs throughout the ventricle and, therefore, does not elicit the same targeted fibrotic, scarring and angiogenic responses, which are all important aspects of the repair to regeneration transition.

It is important to note that following cardiac damage, such as MI (humans) or cryoinjury (zebrafish), the initial phases from injury to repair and scarring are conserved, further strengthening the value of the zebrafish as a powerful tool to understand the limiting factors preventing mammalian regeneration (Fig. 1 and reviewed by Giardoglou and Beis 2019). Both human and zebrafish repair involves an initial inflammatory phase (defined by the recruitment of immune cells and the clearance of cellular debris by phagocytosis) followed by a reparative phase characterised by deposition of collagen and other extracellular matrix (ECM) components and scar formation (Dobaczewski et al. 2011; Chablais and Jazwinska 2012). In humans, this collagenous matrix develops to form a mature scar that is never resolved; however, in zebrafish the deposed collagen is rapidly remodelled and replaced with new myocardium (Chablais et al. 2011; González-Rosa et al. 2011; Schnabel et al. 2011; Hortells et al. 2019).

Cryoinjury results in cell death within the ventricle wall (with apoptotic cells also detectable in the lumen of coronary vessels) which peaks at approximately 4 dpi and decreases progressively to below $0.5 \%$ at $60 \mathrm{dpi}$ (Chablais et al. 2011; González-Rosa et al. 2011; Schnabel et al. 2011). This apoptotic peak is concomitant with the initial inflammatory response and the commencement of neovascularisation with existing coronary vessels sprouting into the injury area (González-Rosa et al. 2011; Marín-Juez et al. 2019). Extensive fibrin accumulation in the injury areas is also seen at 4 dpi (Chablais et al. 2011; Schnabel et al. 2011) but is mostly eliminated by 14-21 dpi (Chablais et al. 2011; González-Rosa et al. 2011; Schnabel et al. 2011). Extensive $\mathrm{CM}$ (and other cell) proliferation is observed during these initial phases of the injury response, peaking within the first week (Chablais et al. 2011; González-Rosa et al. 2011; Schnabel et al. 2011). By $21 \mathrm{dpi}$, vessel coverage of the injured area is complete, and this re-vascularisation of the injured area is so rapid that a mere 40 days after injury the vessels are indistinguishable between controls and injured hearts (González-Rosa et al. 2011; Marín-Juez et al. 2019).

The deposition, remodelling and maturation of the collagen-rich scar are the final phase in the mammalian repair process; however, the resolution of this tissue occurs rapidly in the zebrafish, with scar clearance and regeneration of the cardiac tissue being completed within 60-130 dpi depending on the size of the initial injury (Bevan et al. 2020; Schnabel et al. 2011; Chablais et al. 2011; González-Rosa et al. 2011; and reviewed by Dittrich and Lauridsen 2019). Interestingly, comparative analyses between cryoinjury and resection injury responses indicate differences in the degree of apoptosis, inflammation and scarring, further highlighting variation between injuries (Chablais et al. 2011; Simões et al. 2020). This regenerative capacity in an adult in vivo system provides an unparalleled opportunity to study the molecular and cellular processes of regeneration in an intact environment.

\section{Emerging roles for inflammatory populations}

There has been a recent shift to focus on inflammation as a crucial aspect of the regeneration process. Zebrafish have many innate and adaptive immune cell populations which are analogous to mammals and can be found in similar ratios within the healthy and injured zebrafish heart (Herbomel et al. 1999; Wittamer et al. 2011; Dee et al. 2016; Bevan et al. 


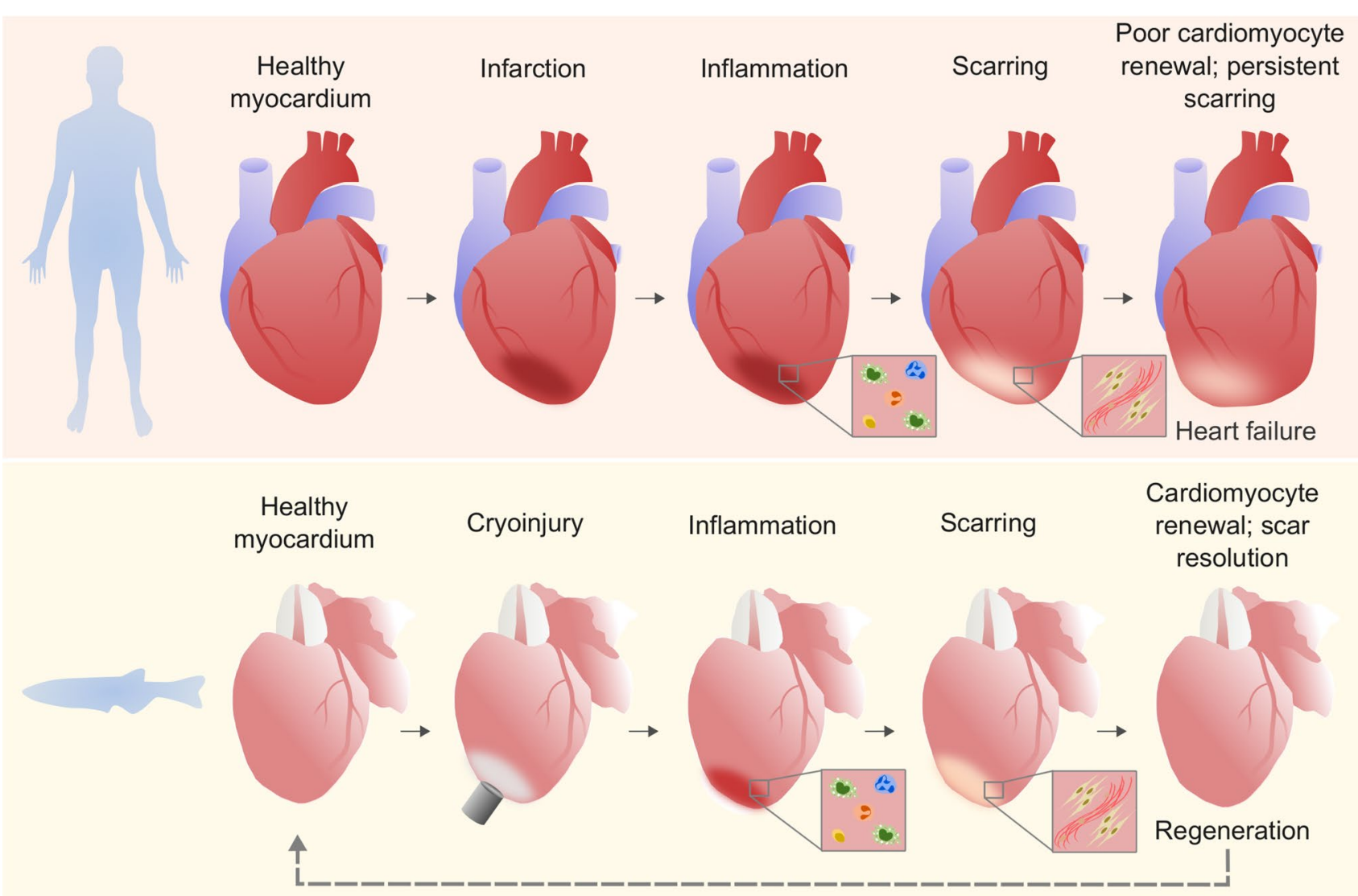

Fig. 1 Human and zebrafish heart repair. Phases of repair/regeneration in human and zebrafish hearts post-injury, showing that initial inflammatory and scarring responses are similar, but the final stages diverge, with humans exhibiting persistent scar tissue and poor

2020). Our group and others have described the systematic recruitment and expansion of innate and adaptive immune cell populations and have begun to uncover their roles in the adult zebrafish heart during the characteristic phases of the repair and regeneration timeline (de Preux Charles et al. 2016; Lai et al. 2017; Hui et al. 2017; Sanz-Morejón et al. 2019; Bevan et al. 2020; Simões et al. 2020) (Fig. 2). This has shown the direct parallels that exist between zebrafish and mammalian cardiac repair, with timely induction and resolution of the inflammatory response being essential to achieve normal regeneration in the zebrafish heart and to regulate many other aspects of repair, including revascularisation, CM proliferation and scar deposition and resolution (Bevan et al. 2020; Simões et al. 2020). Recent observations have also illustrated that without tight regulation of the inflammatory response, excessive immune cell accumulation is inhibitory to regeneration, regardless of the presence of proliferating cells and the ECM (Xu et al. 2019). This demonstrates that inflammatory cells have critical roles in coupling CM proliferation and scar resolution, both of which are central to an efficient regenerative outcome. A thorough renewal of CMs. Zebrafish models of MI (e.g. cryoinjury shown here) exhibit a regenerative phase of scar resolution and CM proliferation, terminating in a return to healthy myocardium

characterisation of immune cell types during all the repair and regeneration stages and a full understanding of the differences between zebrafish and mammals will be essential to understanding their diverse roles after injury. Here we introduce the main immune cell types that have been studied in the context of cardiac regeneration.

\section{Granulocytes}

Granulocytes are key components of the innate immune system and include neutrophils and eosinophils (reviewed by Lin and Loré 2017) whose roles and dynamics following cardiac injury in the adult zebrafish are beginning to be defined (Lai et al. 2017; Xu et al. 2018; Bevan et al. 2020).

Neutrophils are amongst the "first responders" to tissue damage and are rapidly mobilised and recruited to the cryoinjured heart from 6 hours postinjury (hpi) (Bevan et al. 2020). Neutrophil numbers peak during the first $24 \mathrm{hpi}$ (Fig. 2), during which time they are major contributors to the pro-inflammatory phase of repair (Lai et al. 2017; Xu et al. 2018; Bevan et al. 2020). In addition to the phagocytosis 


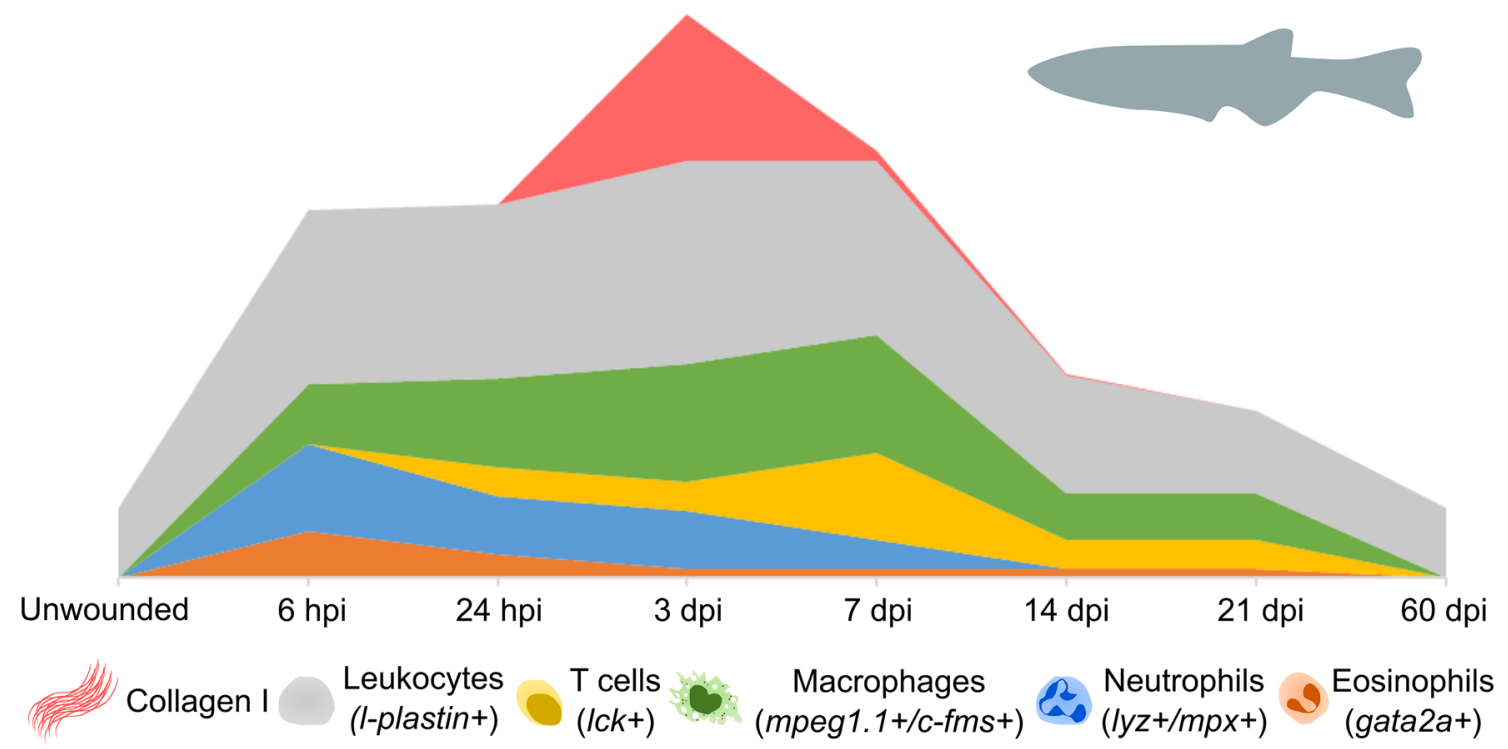

Fig. 2 Immune cell population and ECM dynamics during zebrafish cardiac repair/regeneration. Diagram adapted from data published by Bevan et al. (2020), showing relative waves of immune cell populations and collagen (specifically Collagen I) deposition as detected

of necrotic tissue, recruited neutrophils secrete pro-inflammatory mediators, reactive oxygen species (ROS) and cytokines which recruit additional immune cells and promote myofibroblast differentiation and angiogenesis, which are essential for later stages of regeneration (Lai et al. 2017; $\mathrm{Xu}$ et al. 2018; Bevan et al. 2020). However, the release of ROS by neutrophils, as well as infiltrating monocytes, can cause further tissue necrosis and scarring (Bonaventura et al. 2019). Neutrophil retention has been shown to prolong the inflammatory period leading to delayed scar regression and reduced CM proliferation in heart injury models (Robertson et al. 2014; Lai et al. 2017; Xu et al. 2019), therefore, timely cessation of the neutrophil response is essential for the regenerative outcome observed in zebrafish.

Eosinophils are also rapidly recruited to the injured zebrafish heart and remain elevated from 7-21 dpi (Bevan et al. 2020). Despite this, functional studies have yet to be performed for this cell type in zebrafish so further investigation will be required to elucidate their role in coordinating repair and regeneration.

\section{Macrophages}

The importance of diverse macrophage functions during wound healing has been well-described in both reparative and regenerative organisms (Nahrendorf et al. 2007; Mirza et al. 2009; Lavine et al. 2014; Petrie et al. 2015; NguyenChi et al. 2017; Morales and Allende 2019). This includes phagocytic and pro-inflammatory activity in the initial stages of repair as well as the reversal of the inflammatory over a 60-day time period following cardiac cryoinjury. A colourcoded legend, including markers used to define the relative populations, is shown below the graph

environment to homeostatic conditions (Nguyen-Chi et al. 2017; Bevan et al. 2020). The functional repertoire of macrophages is also ever-expanding, with recent publications identifying novel roles for macrophages in wound angiogenesis (Gurevich et al. 2018), electrical conductance during cardiac homeostasis (Hulsmans et al. 2017) and collagen deposition after heart injury in both murine and zebrafish models (Simões et al. 2020). Macrophages have historically been broadly grouped by function and activation state as either "M1" or "M2", with M1 macrophages thought of as pro-inflammatory and anti-microbial, and M2 macrophages considered to be pro-reparative, with roles in tissue re-modelling, immune regulation, matrix deposition and phagocytosis (reviewed by Lee 2019). However, it is increasingly evident that the diverse, and sometimes surprising, roles being ascribed to cardiac macrophages are coordinated by subsets with highly heterogenous phenotypes, transcriptional profiles and activation states, which are much more transient than the traditional M1 or M2 classification. This M1/M2 nomenclature is now largely seen as an oversimplification, with macrophages more likely to have a spectrum of activation states that inform their function. This plasticity of macrophage phenotype and function is largely controlled by the tissue microenvironment, but is also determined by cellular origin, with tissue resident and monocyte-derived populations shown to be distinct populations (He et al. 2018; Ferrero et al. 2018), which, in turn, is likely to affect their contribution to regeneration and scarring (Lavine et al. 2014).

The genetic tools and imaging capabilities presented by the zebrafish are unveiling important insights into the 
complexities of these populations during adult heart regeneration. Tracking of global macrophage populations during zebrafish heart regeneration indicates that there is an accumulation of macrophages within the ventricle at 3-7 dpi, which is largely resolved by $14 \mathrm{dpi}$ (Fig. 2) (Lai et al. 2017; Bevan et al. 2020; Simões et al. 2020). To date, studies of macrophages in zebrafish have been assisted by the use of select transgenic reporters (namely driven from mpeg1.1 (Ellett et al. 2011) and csflra (Gray et al. 2011) promoters). These can be combined with transgenics that indicate the inflammatory activation of these cells (currently using tnfa (Nguyen-Chi et al. 2017) or illb (Ogryzko et al. 2019) promoters) to further categorise these cells by their inflammatory phenotype. To this end, we have recently shown that distinct waves of macrophage populations (pro-inflammatory and pro-resolutionary) respond to cardiac injury, and interference with these populations leads to prolonged scar retention (Bevan et al. 2020). Similarly, others have shown that delayed monocyte recruitment leads to increased scar area, further demonstrating their requirement for correct injury resolution (Xu et al. 2018). Manipulation of macrophage dynamics also prolongs neutrophil activity and impairs normal neovascularisation, CM replacement and scar removal (De Preux Charles et al. 2016; Lai et al. 2017; Bevan et al. 2020). This suggests that macrophages are important for controlling the inflammatory environment in wounded tissue, partly by neutrophil clearance, and fostering an environment for efficient angiogenesis and CM proliferation.

However, future work is necessary to further delineate the macrophage subsets that are responsible for these processes in zebrafish. Moreover, the recent finding that mpeg1.1, which has been ubiquitously used to label macrophages in zebrafish, is also expressed in a sub-population of B-lymphocytes and natural killer-like cells in adult zebrafish (Moyse and Richardson 2020; Ferrero et al. 2020) demonstrates the need to expand and diversify macrophage markers. Macrophage phenotype and activation states are emerging as key regulators of these opposing processes (Bevan et al. 2020), yet as the importance of the immune response in repair and regeneration becomes clear, the need to fully understand the roles of immune cells in these processes is ever greater. Stratification of these cells will therefore be essential to fully appreciate their contribution to regeneration.

\section{Lymphocytes}

The relationship between the adaptive immune system and regeneration has been a source of debate, as organisms with more advanced immune systems generally have reduced regenerative capacity (Hui et al. 2017; Sattler et al. 2017). As a result, the roles of neutrophils and macrophages in wound healing and regeneration have been extensively studied, yet the contribution of adaptive immune cells is less well understood, particularly with respect to cardiac regeneration. However, it is becoming increasingly apparent that many regenerative organisms, including zebrafish, possess sophisticated adaptive immune systems, justifying the need to study these cells during regeneration.

Lymphocytes of the adaptive immune system, such as $\mathrm{T}$ cells, coordinate antigen-specific immunity. There are many subsets of $\mathrm{T}$ cells with varying functions, such as tumour and viral immunity, cytokine secretion and the establishment of humoral responses. A further subset, CD4+FOXP3+ T regulatory cells (Tregs), are important for suppressing the inflammatory response by secretion of anti-inflammatory cytokines such as IL-10 (Kasheta et al. 2017). These $\mathrm{T}$ cell populations have been shown to have diverse and opposing roles in mouse models of MI, with known functions in wound healing, inflammation and regulation of CM numbers (reviewed by Epelman et al. 2015; Yang et al. 2006; Varda-Bloom et al. 2000); therefore, further investigation is warranted to define $\mathrm{T}$ cell dynamics in response to cardiac injury.

Adult zebrafish possess analogous $\mathrm{T}$ cell populations to the major subsets identified in mammals, namely CD4+ T helper cells (Langenau and Zon 2005; Dee et al. 2016) which include Tregs (Kasheta et al. 2017) and also $\gamma \delta$ $\mathrm{T}$ cells (Wan et al. 2017). They are therefore a fantastic model system to study the role of $\mathrm{T}$ cells in cardiac repair and regeneration. Recently, Hui et al. (2017) described the importance of Tregs in the promotion of CM proliferation and in the control of macrophage activation state postinjury (Hui et al. 2017). Indeed, a comparative study of zebrafish and a closely related but non-regenerative teleost fish, medaka, has highlighted T cell-specific pathways in effective cardiac regeneration in response to cryoinjury. Zebrafish were shown to have strong upregulation of genes involved in $\mathrm{T}$ cell proliferation and B cell receptor signalling unlike medaka (Lai et al. 2017). This suggests that lymphocytes have significant involvement in cardiac repair and regeneration that is yet to be fully elucidated.

Adult zebrafish also possess populations of B cells (Page et al. 2013) and natural killer-like cells (Carmona et al. 2017), and recent studies demonstrate that a proportion of these populations are labelled by mpeg 1.1 transgenics (Moyse and Richardson 2020; Ferrero et al. 2020). Indeed, we have shown that these mpeg 1.1 expressing lymphocytes respond to cardiac cryoinjury in a different temporal manner to mpeg1.1+ macrophages suggesting that they could play a role in repair or regeneration responses (Moyse and Richardson 2020). Future studies will hopefully reveal more about the role these adaptive immune cell populations are playing within a beneficial, regenerative cardiac microenvironment. 


\section{Extracellular matrix composition and function}

The formation of a mature scar which is not resolved majorly inhibits cardiac function post-MI in mammals (reviewed by Hortells et al. 2019) and so the composition of the ECM and the cells which regulate it should also be considered important players in the regenerative process.

The myocardial ECM is an intricate protein network, composed primarily of collagen, and with a tripartite organisation that surrounds CMs (the endomysium), defines major cardiac tissue bundles (the perimysium) and encapsulates the whole cardiac muscle (the epimysium) (reviewed by Frangogiannis 2017). It surrounds the heart, providing a scaffold and maintains the architecture of the tissue. In both mammals and zebrafish, cardiac fibroblasts are the major contributory cell type in ECM production. Normally quiescent, interstitial fibroblasts respond rapidly to stimuli such as injury or hypoxia, re-entering the cell cycle, synthesising ECM proteins, moderating cell-cell communication and producing activated fibroblasts and myofibroblasts (Moore-Morris et al. 2014; Kanisicak et al. 2016; Ivey et al. 2018).

In mammals, the cardiac ECM is thought to be composed mainly of collagen I (> 85\%), with other collagens, fibronectin, glycosaminoglycans (GAGs) and proteoglycans comprising the rest of the matrix (reviewed by Frangogiannis 2017). The exact composition of the zebrafish ECM is unknown; however, comparison with murine samples has revealed that zebrafish ECM contains significantly less collagen and more elastins and GAGs (Chen et al. 2016). Additional information on the ECM composition has been gleaned from immunodetection assays, which have shown the presence of several matrix proteins during zebrafish cardiac regeneration, including structural proteins such as non-fibrillar Collagen XII and fibrillar Collagen I, the adhesive protein Fibronectin and the deadhesive protein Tenascin C (Chablais and Jazwinska 2012; Wang et al. 2013; Marro et al. 2016). Decellularised cardiac ECM from zebrafish has been shown to have a significant proliferative effect on human cardiac precursor cells and enables endogenous regeneration of mouse heart tissue post-MI (Chen et al. 2016). These clear differences in zebrafish vs mammalian ECM highlight its importance in mediating regeneration and demonstrate the merit in continued research into the exact manner in which ECM components are generated and resolved.

In adult mammals, cardiac fibroblasts are required for scar formation, and their contribution to the ECM profile post-injury can influence CM proliferation and hypertrophy, but ultimately there is low CM proliferation, limited scar resolution and thus fibroblasts transition to matrifibrocytes and a mature scar is formed that reduces cardiac function (reviewed by Hortells et al. 2019). However, in zebrafish, endocardial fibroblasts synthesise ECM collagen post-injury and are inactivated during scar resolution. Though limiting the fibrotic response via ablation of colla2-expressing cells does not affect regeneration, it does reduce the number of proliferating CMs, hence both fibroblasts and the ECM are necessary to stimulate CM proliferation and regeneration (Sánchez-Iranzo et al. 2018). Recent work has also revealed that it is not only myofibroblasts that contribute to fibrosis during heart repair, but macrophages also deposit collagen post-injury in both zebrafish and mouse models (Simões et al. 2020). This newly identified function for macrophages further highlights the fascinating potential of immune cells, and also the extent to which their importance is still incompletely understood.

\section{Extracellular vesicles, cilia and signalling networks}

While looking outside of the traditional focus on CM proliferation, it is important to consider not just which cell types are implicated, but how they are recruited and activated. One under-explored aspect of cell-cell communication is the topical field of extracellular vesicle (EV) research. These particles, bound by a lipid bi-layer, are produced and released by most cell types and can be broadly divided into three classes depending on their biogenesis: exosomes, microvesicles and apoptotic bodies (Van Niel et al. 2018; Caruso and Poon 2018). EVs have been isolated from pericardial fluid from patients undergoing cardiac surgery (Kuosmanen et al. 2015; Beltrami et al. 2017) and have been implicated in the progression of cardiovascular disease (Wang et al. 2014; Emanueli et al. 2015), but are also thought to have roles in mediating cell-cell communication between different cell types within the heart, including CMs, fibroblasts and immune cells (Bang et al. 2014, 2015; Todorova et al. 2017; Zhou et al. 2019). Further to this, there is emerging data that suggest there may be EV populations that are cardioprotective and promote angiogenesis (Emanueli et al. 2015; Todorova et al. 2017; Zhou et al. 2019).

Despite this mounting evidence showing the importance of EVs, what we know thus far has been largely derived from in vitro studies (Van Niel et al. 2018); however, novel methods are being developed to address the complexity and heterogeneity of endogenous EVs. Our laboratory has recently developed one such tool, combining the in vivo nature of the zebrafish model with a cell-membrane tagged fluorophore approach to facilitate future investigations of endogenous cell-type-specific EV populations (Scott et al. 2019). This approach coupled with the cardiac cryoinjury model 
demonstrates dynamic changes in EV production post-injury and has potential to unlock the role of EVs in cell communication during repair and regeneration.

Another sub-cellular component with potential implications in this area of cell-cell communication is the cilium. Research into the primary cilium and its role in different disease states has also expanded exponentially in recent years. Initially thought to be no more than an evolutionary vestige, primary cilia are now known to be present on the majority of cells and have been revealed to be crucial signalling antennae, sending and receiving information across cell and tissue types. Primary cilia have roles in fundamental cell processes like proliferation, differentiation and regulation of the cell cycle (Delling et al. 2013; Yuan et al. 2016) and respond to a wide range of stimuli including growth factors (Schneider et al. 2005) and glucocorticoids (Wang et al. 2012) as well as mechanical stress and flow (Hierck et al. 2008; Nauli et al. 2013). It is thus unsurprising that cilia are becoming a subject of interest in the field of cardiovascular research, with a recent publication detailing their previously unknown requirement for cardiac fibrosis following MI in both zebrafish and human tissue (Villalobos et al. 2019).

Sub-cellular components such as cilia and EVs have historically been challenging to study due to limitations in imaging and analysis; however, novel methods and improved technology are revealing that these miniscule actors play crucial roles in regulating complex and dynamic microenvironments in vivo and new studies are highlighting the zebrafish as an ideal model system in which to study them.

During regeneration, CM proliferation must proceed in concert with the removal of the deposited scar tissue. The damaged tissue is subject to remodelling and re-organisation as it grows, and these processes are orchestrated via a combination of adhesion, migration and signalling events (reviewed by Sanz-Morejón and Mercader 2020). The roles and consequences of signalling pathways in these processes are complex and manifold, with CMs at the injury site exposed to signals including Pdgf, RA, Igf, Shh, Tgf $\beta$ ligands, BMP and $\mathrm{Nrg} 1$, which are secreted from the epicardium, epicardium-derived cells, endocardial cells and circulating cells (reviewed by González-Rosa et al. 2017). $\mathrm{NF}-\kappa \mathrm{B}$ signalling is also required for $\mathrm{CM}$ dedifferentiation and proliferation, as well as epicardial regeneration (Karra et al. 2015), and Notch signalling (via serpine 1) is required for endocardial and myocardial proliferation (Münch et al. 2017). Hedgehog signalling ligands have been determined to be essential for controlling epicardial migration and are produced by smooth muscle cells from the bulbus arteriosus (Wang et al. 2015). The important publications in this area are so numerous that a comprehensive discussion of these processes goes beyond the focus of this publication and have been discussed recently elsewhere (González-Rosa et al. 2017). However, the versatility of the zebrafish model has led to the identification of important loci in the control of scar deposition/resolution and other regenerative processes, and so some specific examples of newly-identified genetic players and their contributions to the understanding of the complex signalling cascades required for regeneration are featured here.

It has recently been shown that the transcription factor Runx 1 is a key regulator of both scar deposition and degradation, as well as proliferation of the myocardium (Koth et al. 2020). Not only was Runx 1 seen to be specifically upregulated in endocardial cells and thrombocytes in the injury region, which in turn induced expression of smooth muscle and collagen genes during zebrafish heart regeneration, but targeted mutation and the subsequent absence of runxl resulted in an increase in survival and proliferation of the myocardium and overall heart regeneration and decreased fibrosis resulting from a reduction in myofibroblast formation and upregulation of the fibrin degeneration pathway (Koth et al. 2020). This discovery is particularly interesting given that Runx 1 is upregulated in CMs postinjury in a number of species, both regenerative and not, and presents a potential therapeutic target that could be manipulated to induce endogenous human heart regeneration (Gattenlöhner et al. 2003; Kubin et al. 2011; Eulalio et al. 2012; Górnikiewicz et al. 2016; Goldman et al. 2017; Koth et al. 2020).

In terms of $\mathrm{CM}$ proliferation, there have also been some interesting observations that help set the process apart in zebrafish, identifying differences in the effects of signalling mechanisms. Increased Yap/Taz in murine CMs has been shown to augment the renewal of cardiac tissue following ischaemia, yet CM proliferation in zebrafish yap mutants is not altered following cryoinjury, contrary to reports from the murine study; however, the collagen composition of scars is decreased and the injury size observed is larger, indicating that Yap is required for scar formation during zebrafish heart repair, indirectly mediating ECM deposition (Leach et al. 2017). The role of nerve cells in CM proliferation is both conserved and disparate between the regenerative models of zebrafish and neonatal mice. Cardiac re-innervation following injury is necessary in both neonatal mice and zebrafish, with Nrg1 capable of stimulating CM proliferation after injury (Mahmoud et al. 2015). Interestingly, however, the source of $\mathrm{Nrg} 1$ is not conserved, as murine production of $\mathrm{Nrg} 1$ occurs in nerve cells but it derives from perivascular cells in zebrafish, despite the conserved requirement for nerve cells in both systems (Gemberling et al. 2015; Mahmoud et al. 2015). Exploration of these differences between regenerative and non-regenerative, in addition to vertebrate and invertebrate species may be key to finding targets for future genetic or pharmaceutical therapies. A study of the revascularisation that occurs post-injury has also revealed that there are two kinds of coronary sprouting 
that have separate mechanistic controls, with superficial sprouting (within the regenerating epicardium) regulated by chemokines cxcl12 and cxcr4, whereas intraventricular sprouting (towards the activated endocardium) is controlled by vegfa. These then combine to provide a scaffold for $\mathrm{CM}$ repopulation of the injured heart; however, inhibition of early revascularisation via a dominant negative form of vegfaa reduces $\mathrm{CM}$ proliferation and inhibits regeneration (Marín-Juez et al. 2019). Consistently, knockout of cxcr4a also inhibits regeneration (Harrison et al. 2015). This type of data can help compare and highlight important differences in the regulatory pathways that are controlling the regenerative response.

This cache of valuable genetic data is not limited to zebrafish however, as recent studies in another teleost fish have shown. Comparative genetics in a study of Mexican cave fish Astyanax mexicanus has also yielded a wealth of data, following the striking observation that a surface-dwelling population of these fish are capable of complete cardiac regeneration after injury yet their cave-dwelling counterparts are not, despite being of the same species. Independent evolution of the populations has provided an unparalleled system for direct comparison of their respective scarring and regenerative responses, and the ability to identify key genetic players in the capacity for heart regeneration without the complications of presented by interspecies comparisons. The authors were able to identify lrrcl0 as a gene specifically upregulated in surface fish (regenerative) compared to cavefish (non-regenerative), and a complementary zebrafish knockout model showed impaired heart regeneration despite unaffected proliferation of the CMs (Stockdale et al. 2018). This further supports the importance of looking beyond CM proliferation to consider all the components of the complex microenvironment. Further to this, quantitative trait analysis allowed the authors to identify three genomic loci that seem to be linked to the magnitude of heart regeneration (Stockdale et al. 2018).

Though regeneration mechanisms are generally believed to be tissue-specific (reviewed by Beffagna 2019), there is also evidence from zebrafish to suggest that there may in fact be some common regulatory elements, with the discovery by Pfefferli and Jaźwińska of careg, a regulatory element that contains a ctgfa upstream sequence that is transiently activated but common to both heart and fin regeneration, and induced by TGF- $\beta /$ Activin- $\beta$ signalling (Pfefferli and Jaźwińska 2017).

Though in no way comprehensive, the above-mentioned studies show how the zebrafish can provide valuable insights into the signalling pathways and communication networks involved in a regenerative vertebrate system. By comparing these to non-regenerative systems, this will help to elucidate conserved regulatory elements but also identify key differences that may be critical to achieving a regenerative phenotype.

\section{Looking beyond cardiomyocytes}

As we look beyond CMs, the adverse microenvironment is full of complex cues that are not yet fully understood. The cells of the immune response alone have an ever-increasing functional roster in both repair and regeneration, including emerging roles for macrophages in wound angiogenesis (Gurevich et al. 2018) and collagen deposition (Simões et al. 2020). Additionally, studies are describing multiple crucial roles for other cell types within the heart including epicardial cells (reviewed by Masters and Riley 2014; Cao and Poss 2018), endothelial cells (Marín-Juez et al. 2016), nerves (Mahmoud et al. 2015), fibroblasts (Sánchez-Iranzo et al. 2018) and lymphatic cells (Gancz et al. 2019; Harrison et al. 2019; Vivien et al. 2019) (summarised in Fig. 3 and Table 1). The importance of all the steps in the cascade should not be underestimated, as each additional piece of the puzzle contributes to rebuilding the heart. The interplay between the physical and molecular processes that orchestrate to regenerate the heart is still incompletely understood; however, the zebrafish presents an invaluable system to study the interplay of these different cells and processes. This regenerative adult in vivo model is amenable to highthroughput genetic editing approaches and live-imaging is unparalleled in its potential to unravel the complexities of the therapeutic holy grail that is human heart regeneration.

\section{Summary}

In this review, we have covered the advantages of the zebrafish model for cardiac regeneration research with a particular focus on the roles of different cell types within the cardiac microenvironment. Many of these roles are being uncovered by studying zebrafish and their remarkable natural regenerative ability. There is no doubt that the recovery of CM number is crucial to the regeneration of the heart and the return to full functional capacity, but studies in zebrafish and other regenerative models are revealing the plethora of signalling and other functions delivered by immune cells, epicardial cells, nerves, fibroblasts and endothelial cells that are crucial to supporting this regenerative outcome. In our minds, future therapeutic approaches will need to incorporate the protection or replacement of these other cell types, as well as CMs, to be effective. Groups studying the zebrafish are leading the way in this investigation into the complex cardiac microenvironment. 


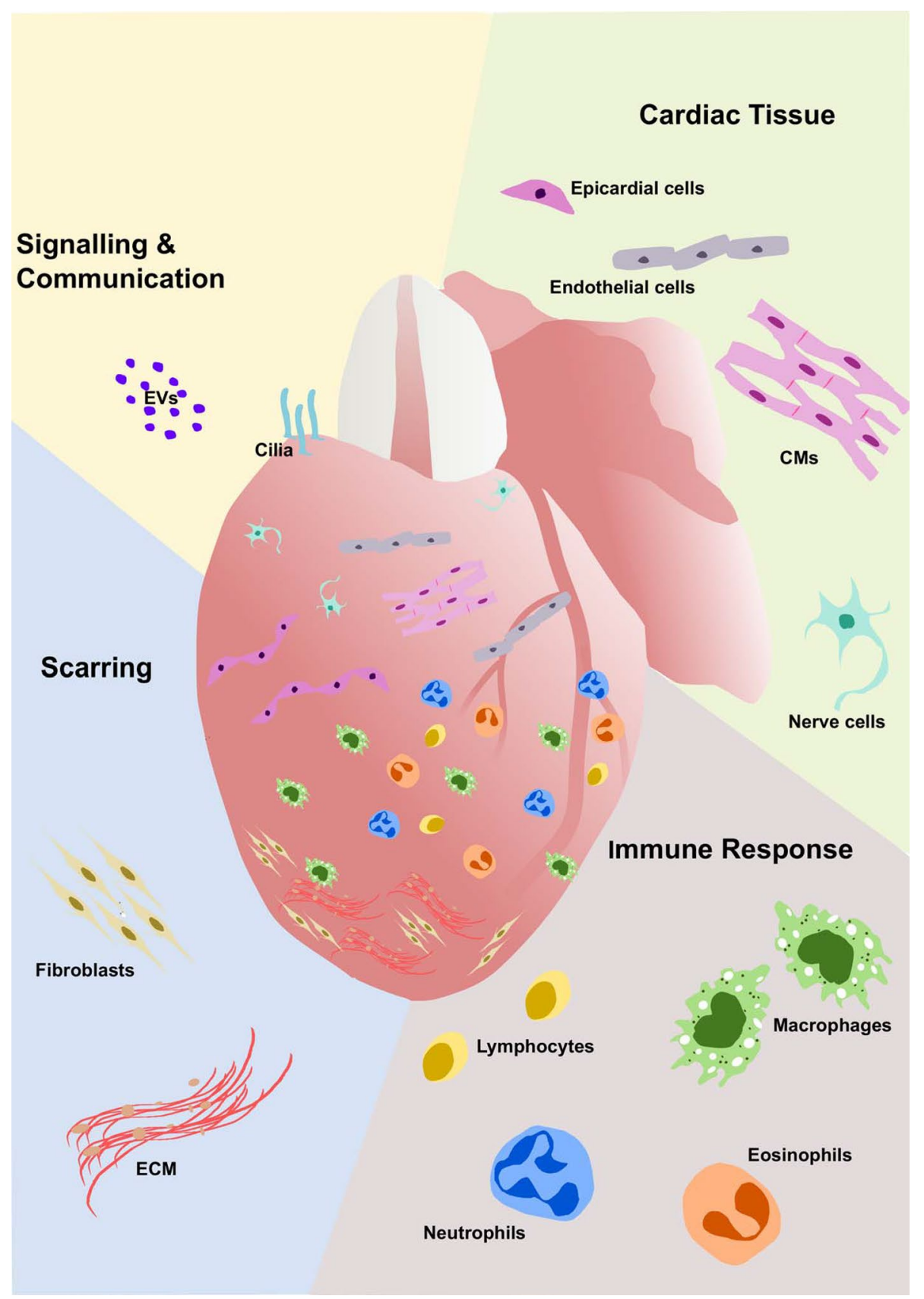

Fig. 3 Heart repair and regeneration requires a concerted effort by many different cell types. Representation of the numerous cell populations required throughout heart repair and regeneration in the adult zebrafish. Sample publications detailing the roles of these different cell types are listed in Table 1 
Table 1 Components of the cardiac microenvironment important for regeneration

Immune cells

Macrophages

Neutrophils

Eosinophils

Lymphocytes

Cardiac tissue

Cardiomyocytes

Fibroblasts

Endothelial/endocardial cells

Epicardial cells

Nerve cells

Lymphatic cells

Scarring

ECM

\section{Cilia and extracellular vesicles}

EVs

Cilia
Bevan et al. (2020); Ellett et al. (2011); Ferrero et al. (2018); Gray et al. (2011); He et al. (2018); Lai et al. (2017); Lavine et al. (2014); Nguyen-Chi et al. (2017); Ogryzko et al. (2019); de Preux Charles et al. (2016); Simões et al. (2020); Xu et al. (2018)

Bevan et al. (2020); Lai et al. (2017); Robertson et al. (2014); Xu et al. (2018)

Bevan et al. (2020)

Bevan et al. (2020); Carmona et al. (2017); Dee et al. (2016); Hui et al. (2017); Kasheta et al. (2017); Lai et al. (2017); Langenau and Zon, (2005); Moyse and Richardson (2020); Page et al. 2013; Wan et al. (2017)

Bise et al. (2020); Chablais et al. (2011); González-Rosa et al. (2011); González-Rosa et al. (2018); Jopling et al. (2010); Kikuchi et al. (2010); *Kikuchi, (2015); Schnabel et al. (2011)

Sánchez-Iranzo et al. (2018)

Münch et al. (2017); Marín-Juez et al. (2019)

*Cao and Poss (2018); Marín-Juez et al. (2019); *Masters and Riley (2014); Schnabel et al. (2011); Wang et al. (2013); Wang et al. (2015)

Mahmoud et al. (2015)

Gancz et al. (2019); Harrison et al. (2019); Vivien et al. (2019)

Chablais and Jaźwińska (2012); Chen et al. (2016); Marro et al. (2016); Sánchez-Iranzo et al. (2018); Simões et al. (2020); Wang et al. (2013)

Scott et al. (2019)

Villalobos et al. (2019)

Sample publications detailing the role of various important components of the microenvironment required for zebrafish heart regeneration are listed here. This table is not exhaustive but includes some of the major publications discussed throughout this review

An asterisk (*) denotes review articles

Author contributions RRy conceptualised ideas, wrote the manuscript and created the figures, BM wrote the manuscript and assisted in figure creation and RRi conceptualised ideas and wrote the manuscript.

Funding This work was supported by the BHF Oxbridge Centre of Regenerative Medicine (RM/17/2/33380), a BHF Intermediate Fellowship to RRi (FS/15/2/31225) and a Wellcome Trust funded PhD studentship to BM $(108907 / \mathrm{Z} / 15 / \mathrm{Z})$.

\section{Compliance with ethical standards}

Conflict of interest The authors declare no competing interests.

Consent for publication All authors consent to publication.

Open Access This article is licensed under a Creative Commons Attribution 4.0 International License, which permits use, sharing, adaptation, distribution and reproduction in any medium or format, as long as you give appropriate credit to the original author(s) and the source, provide a link to the Creative Commons licence, and indicate if changes were made. The images or other third party material in this article are included in the article's Creative Commons licence, unless indicated otherwise in a credit line to the material. If material is not included in the article's Creative Commons licence and your intended use is not permitted by statutory regulation or exceeds the permitted use, you will need to obtain permission directly from the copyright holder. To view a copy of this licence, visit http://creativecommons.org/licenses/by/4.0/.

\section{References}

Ahuja P (2004) Sequential myofibrillar breakdown accompanies mitotic division of mammalian cardiomyocytes. J Cell Sci 117:3295-3306. https://doi.org/10.1242/jcs.01159

Alkass K, Panula J, Westman M et al (2015) No evidence for cardiomyocyte number expansion in preadolescent mice. Cell 163:10261036. https://doi.org/10.1016/j.cell.2015.10.035

Bang C, Antoniades C, Antonopoulos AS et al (2015) Intercellular communication lessons in heart failure. Eur J Heart Fail 17:1091-1103. https://doi.org/10.1002/ejhf.399

Bang C, Batkai S, Dangwal S et al (2014) Cardiac fibroblast-derived microRNA passenger strand-enriched exosomes mediate cardiomyocyte hypertrophy. J Clin Invest 124:2136-2146. https ://doi.org/10.1172/JCI70577

Beffagna G (2019) Zebrafish as a smart model to understand regeneration after heart injury: how fish could help humans. Front Cardiovasc Med 6:107. https://doi.org/10.3389/fcvm.2019.00107

Bellayr I, Mu X, Li Y (2009) Biochemical insights into the role of matrix metalloproteinases in regeneration: challenges and recent developments. Future Med Chem 1:1095-1111. https:// doi.org/10.4155/fmc. 09.83

Beltrami C, Besnier M, Shantikumar S et al (2017) Human pericardial fluid contains exosomes enriched with cardiovascularexpressed MicroRNAs and promotes therapeutic angiogenesis. Mol Ther 25:679-693. https://doi.org/10.1016/j.ymthe .2016 .12 .022

Bergmann O, Bhardwaj RD, Bernard S et al (2009) Evidence for cardiomyocyte renewal in humans. Science 324(5923):98-102. https ://doi.org/10.1126/science.1164680 
Bevan L, Lim ZW, Venkatesh B et al (2020) Specific macrophage populations promote both cardiac scar deposition and subsequent resolution in adult zebrafish. Cardiovasc Res 116:1357-1371. https://doi.org/10.1093/cvr/cvz221

Bise T, Sallin P, Pfefferli C, Jaźwińska A (2020) Multiple cryoinjuries modulate the efficiency of zebrafish heart regeneration. Sci Rep 10:11551. https://doi.org/10.1038/s41598-020-68200-1

Bonaventura A, Montecucco F, Dallegri F et al (2019) Novel findings in neutrophil biology and their impact on cardiovascular disease. Cardiovasc Res 115:1266-1285. https://doi.org/10.1093/ cvr/cvz084

Braunwald E (2015) The war against heart failure: the Lancet lecture. Lancet 385:812-824. https://doi.org/10.1016/S0140 $-6736(14) 61889-4$

Bryant DM, O'Meara CC, Ho NN et al (2015) A systematic analysis of neonatal mouse heart regeneration after apical resection. $\mathrm{J}$ Mol Cell Cardiol 79:315-318. https://doi.org/10.1016/j.yjmcc .2014.12.011

Cahill TJ, Choudhury RP, Riley PR (2017) Heart regeneration and repair after myocardial infarction: translational opportunities for novel therapeutics. Nat Rev Drug Discov 16:699-717. https ://doi.org/10.1038/nrd.2017.106

Cao J, Poss KD (2018) The epicardium as a hub for heart regeneration. Nat Rev Cardiol 15:631-647. https://doi.org/10.1038/ s41569-018-0046-4

Carmona SJ, Teichmann SA, Ferreira L et al (2017) Single-cell transcriptome analysis of fish immune cells provides insight into the evolution of vertebrate immune cell types. Genome Res 27:451-461. https://doi.org/10.1101/gr.207704.116

Caruso S, Poon IKH (2018) Apoptotic cell-derived extracellular vesicles: more than just debris. Front Immunol 9:1486. https ://doi.org/10.3389/fimmu.2018.01486

Chablais F, Jazwinska A (2012) The regenerative capacity of the zebrafish heart is dependent on TGF signaling. Development 139:1921-1930. https://doi.org/10.1242/dev.078543

Chablais F, Veit J, Rainer G, Jaźwińska A (2011) The zebrafish heart regenerates after cryoinjury-induced myocardial infarction. BMC Dev Biol 11:21. https://doi. org/10.1186/1471-213X-11-21

Chen K, Huang Y, Singh R, Wang ZZ (2020) Arrhythmogenic risks of stem cell replacement therapy for cardiovascular diseases. J Cell Physiol 235:6257-6267. https://doi.org/10.1002/jcp.29554

Chen WCW, Wang Z, Missinato MA et al (2016) Decellularized zebrafish cardiac extracellular matrix induces mammalian heart regeneration. Sci Adv 2:e1600844. https://doi.org/10.1126/ sciadv. 1600844

de Preux Charles A-S, Bise T, Baier F et al (2016) Distinct effects of inflammation on preconditioning and regeneration of the adult zebrafish heart. Open Biol 6:160102. https://doi.org/10.1098/ rsob. 160102

Dee CT, Nagaraju RT, Athanasiadis EI et al (2016) CD4-transgenic zebrafish reveal tissue-resident Th2- and regulatory T cell-like populations and diverse mononuclear phagocytes. J Immunol 197:3520-3530. https://doi.org/10.4049/jimmunol.1600959

Delling M, Decaen PG, Doerner JF et al (2013) Primary cilia are specialized calcium signalling organelles. Nature 504(7479):311314. https://doi.org/10.1038/nature12833

Dittrich A, Lauridsen H (2019) Myocardial infarction and the immune response-scarring or regeneration? A comparative look at mammals and popular regenerating animal models. J Immunol Regen Med 4:100016. https://doi.org/10.1016/j.regen .2019 .100016

Dobaczewski M, Chen W, Frangogiannis NG (2011) Transforming growth factor (TGF)- $\beta$ signaling in cardiac remodeling. $J$ Mol Cell Cardiol 51:600-606. https://doi.org/10.1016/j.yjmcc .2010 .10 .033
Dooley K (2000) Zebrafish: a model system for the study of human disease. Curr Opin Genet Dev 10:252-256. https://doi.org/10.1016/ S0959-437X(00)00074-5

Ellett F, Pase L, Hayman JW et al (2011) mpeg1 promoter transgenes direct macrophage-lineage expression in zebrafish. Blood 117:e49-e56. https://doi.org/10.1182/blood-2010-10-314120

Emanueli C, Shearn AIU, Angelini GD, Sahoo S (2015) Exosomes and exosomal miRNAs in cardiovascular protection and repair. Vascul Pharmacol 71:24-30. https://doi.org/10.1016/j. vph.2015.02.008

Epelman S, Liu PP, Mann DL (2015) Role of innate and adaptive immune mechanisms in cardiac injury and repair. Nat Rev Immunol 15:117-129. https://doi.org/10.1038/nri3800

Eulalio A, Mano M, Ferro MD et al (2012) Functional screening identifies miRNAs inducing cardiac regeneration. Nature 492:376381. https://doi.org/10.1038/nature11739

Ferrero G, Gomez E, Lyer S et al (2020) The macrophage-expressed gene ( mpeg ) 1 identifies a subpopulation of B cells in the adult zebrafish. J Leukoc Biol 107:431-443. https://doi.org/10.1002/ JLB.1A1119-223R

Ferrero G, Mahony CB, Dupuis E et al (2018) Embryonic microglia derive from primitive macrophages and are replaced by cmybdependent definitive microglia in zebrafish. Cell Rep 24:130141. https://doi.org/10.1016/j.celrep.2018.05.066

Frangogiannis NG (2017) The extracellular matrix in myocardial injury, repair, and remodeling. J Clin Invest 127:1600-1612. https://doi.org/10.1172/JCI87491

Gancz D, Raftrey BC, Perlmoter G et al (2019) Distinct origins and molecular mechanisms contribute to lymphatic formation during cardiac growth and regeneration. Elife 8:e44153. https://doi. org/10.7554/eLife.44153

Gattenlöhner S, Waller C, Ertl G et al (2003) NCAM(CD56) and RUNX1(AML1) are up-regulated in human ischemic cardiomyopathy and a rat model of chronic cardiac ischemia. Am J Pathol 163:1081-1090. https://doi.org/10.1016/S0002-9440(10)63467-0

Gemberling M, Karra R, Dickson AL, Poss KD (2015) Nrg1 is an injury-induced cardiomyocyte mitogen for the endogenous heart regeneration program in zebrafish. Elife 4:e05871. https://doi. org/10.7554/eLife.05871

Giardoglou P, Beis D (2019) On zebrafish disease models and matters of the heart. Biomedicines 7(1):15

Goldman JA, Kuzu G, Lee N et al (2017) Resolving heart regeneration by replacement histone profiling. Dev Cell 40(4):392-404. e5. https://doi.org/10.1016/j.devcel.2017.01.013

González-Rosa JM, Burns CE, Burns CG (2017) Zebrafish heart regeneration: 15 years of discoveries. Regeneration 44(4):433446.e7. https://doi.org/10.1002/reg2.83

Gonzalez-Rosa JM, Martin V, Peralta M et al (2011) Extensive scar formation and regression during heart regeneration after cryoinjury in zebrafish. Development 138:1663-1674. https://doi. org/10.1242/dev.060897

González-Rosa JM, Sharpe M, Field D et al (2018) Myocardial polyploidization creates a barrier to heart regeneration in zebrafish. Dev Cell 44:433-446.e7. https://doi.org/10.1016/j. devcel.2018.01.021

Górnikiewicz B, Ronowicz A, Krzemiński M, Sachadyn P (2016) Changes in gene methylation patterns in neonatal murine hearts: implications for the regenerative potential. BMC Genom 17:231. https://doi.org/10.1186/s12864-016-2545-1

Gray C, Loynes C, Whyte M et al (2011) Simultaneous intravital imaging of macrophage and neutrophil behaviour during inflammation using a novel transgenic zebrafish. Thromb Haemost 105:811-819. https://doi.org/10.1160/TH10-08-0525

Gurevich DB, Severn CE, Twomey C et al (2018) Live imaging of wound angiogenesis reveals macrophage orchestrated vessel 
sprouting and regression. EMBO J 37:e97786. https://doi. org/10.15252/embj.201797786

Harrison MR, Feng X, Mo G et al (2019) Late developing cardiac lymphatic vasculature supports adult zebrafish heart function and regeneration. Elife 8:e42762. https://doi.org/10.7554/eLife .42762

Harrison MRM, Bussmann J, Huang Y et al (2015) Chemokineguided angiogenesis directs coronary vasculature formation in zebrafish. Dev Cell 33(4):442-454. https://doi.org/10.1016/j. devcel.2015.04.001

Hashimoto H, Olson EN, Bassel-Duby R (2018) Therapeutic approaches for cardiac regeneration and repair. Nat Rev Cardiol 15:585-600. https://doi.org/10.1038/s41569-018-0036-6

He S, Chen J, Jiang Y et al (2018) Adult zebrafish Langerhans cells arise from hematopoietic stem/progenitor cells. Elife 7:e36131. https://doi.org/10.7554/eLife.36131

Herbomel P, Thisse B, Thisse C (1999) Ontogeny and behaviour of early macrophages in the zebrafish embryo. Development 126(17):3735-3745

Hierck BP, Van der Heiden K, Alkemade FE et al (2008) Primary cilia sensitize endothelial cells for fluid shear stress. Dev Dyn 237:725-735. https://doi.org/10.1002/dvdy.21472

Hirose K, Payumo AY, Cutie S et al (2019) Evidence for hormonal control of heart regenerative capacity during endothermy acquisition. Science 364(6436):184-188. https://doi. org/10.1126/science.aar2038

Hortells L, Johansen AKZ, Yutzey KE (2019) Cardiac fibroblasts and the extracellular matrix in regenerative and nonregenerative hearts. J Cardiovasc Dev Dis 6:29. https://doi.org/10.3390/ jedd6030029

Howe K, Clark MD, Torroja CF et al (2013) The zebrafish reference genome sequence and its relationship to the human genome. Nature 496:498-503. https://doi.org/10.1038/nature12111

Hui SP, Sheng DZ, Sugimoto K et al (2017) Zebrafish regulatory T cells mediate organ-specific regenerative programs. Dev Cell 43:659-672.e5. https://doi.org/10.1016/j.devcel.2017.11.010

Hulsmans M, Clauss S, Xiao L et al (2017) Macrophages facilitate electrical conduction in the heart. Cell 169:510-522.e20. https ://doi.org/10.1016/j.cell.2017.03.050

Ivey MJ, Kuwabara JT, Pai JT et al (2018) Resident fibroblast expansion during cardiac growth and remodeling. J Mol Cell Cardiol 114:161-174. https://doi.org/10.1016/j.yjmcc.2017.11.012

Jopling C, Sleep E, Raya M et al (2010) Zebrafish heart regeneration occurs by cardiomyocyte dedifferentiation and proliferation. Nature 464:606-609. https://doi.org/10.1038/nature08899

Kanisicak O, Khalil H, Ivey MJ et al (2016) Genetic lineage tracing defines myofibroblast origin and function in the injured heart. Nat Commun 7:12260. https://doi.org/10.1038/ncomms12260

Karra R, Knecht AK, Kikuchi K, Poss KD (2015) Myocardial NF-кB activation is essential for zebrafish heart regeneration. Proc Natl Acad Sci USA 112(43):13255-13260. https://doi.org/10.1073/ pnas. 1511209112

Kasheta M, Painter CA, Moore FE et al (2017) Identification and characterization of $\mathrm{T}$ reg-like cells in zebrafish. J Exp Med 214:3519-3530. https://doi.org/10.1084/jem.20162084

Kikuchi K (2015) Dedifferentiation, transdifferentiation, and proliferation: mechanisms underlying cardiac muscle regeneration in zebrafish. Curr Pathobiol Rep 3:81-88. https://doi.org/10.1007/ s40139-015-0063-5

Kikuchi K, Holdway JE, Werdich AA et al (2010) Primary contribution to zebrafish heart regeneration by gata4+ cardiomyocytes. Nature 464:601-605. https://doi.org/10.1038/nature08804

Koster R, Sassen WA (2015) A molecular toolbox for genetic manipulation of zebrafish. Adv Genom Genet 5:151-163. https://doi. org/10.2147/AGG.S57585
Koth J, Wang X, Killen AC et al (2020) Runx1 promotes scar deposition and inhibits myocardial proliferation and survival during zebrafish heart regeneration. Development. https://doi. org/10.1242/dev.186569

Kubin T, Pöling J, Kostin S et al (2011) Oncostatin M is a major mediator of cardiomyocyte dedifferentiation and remodeling. Cell Stem Cell 9:420-432. https://doi.org/10.1016/j.stem.2011.08.013

Kuosmanen SM, Hartikainen J, Hippeläinen M et al (2015) MicroRNA profiling of pericardial fluid samples from patients with heart failure. PLoS ONE 10:e0119646. https://doi.org/10.1371/journ al.pone. 0119646

Lai S-L, Marín-Juez R, Moura PL et al (2017) Reciprocal analyses in zebrafish and medaka reveal that harnessing the immune response promotes cardiac regeneration. Elife 6:e25605. https:// doi.org/10.7554/eLife. 25605

Langenau DM, Zon LI (2005) The zebrafish: a new model of T-cell and thymic development. Nat Rev Immunol 5:307-317. https:// doi.org/10.1038/nri1590

Lavine KJ, Epelman S, Uchida K et al (2014) Distinct macrophage lineages contribute to disparate patterns of cardiac recovery and remodeling in the neonatal and adult heart. Proc Natl Acad Sci USA 111(45):16029-16034. https://doi.org/10.1073/pnas.14065 08111

Leach JP, Heallen T, Zhang M et al (2017) Hippo pathway deficiency reverses systolic heart failure after infarction. Nature 550:260264. https://doi.org/10.1038/nature24045

Lee KY (2019) M1 and M2 polarization of macrophages: a minireview. Med Biol Sci Eng 2:1-5. https://doi.org/10.30579/ mbse.2019.2.1.1

Lieschke GJ, Currie PD (2007) Animal models of human disease: zebrafish swim into view. Nat Rev Genet 8:353-367. https://doi. org/10.1038/nrg2091

Lin A, Loré K (2017) Granulocytes: new members of the antigenpresenting cell family. Front Immunol 8:1781. https://doi. org/10.3389/fimmu.2017.01781

Liu Y-W, Chen B, Yang X et al (2018) Human embryonic stem cellderived cardiomyocytes restore function in infarcted hearts of non-human primates. Nat Biotechnol 36:597-605. https://doi. org/10.1038/nbt.4162

Mahmoud AI, O'Meara CC, Gemberling M et al (2015) Nerves regulate cardiomyocyte proliferation and heart regeneration. Dev Cell 34:387-399. https://doi.org/10.1016/j.devcel.2015.06.017

Marín-Juez R, El-Sammak H, Helker CSM et al (2019) Coronary revascularization during heart regeneration is regulated by epicardial and endocardial cues and forms a scaffold for cardiomyocyte repopulation. Dev Cell 51:503-515.e4. https://doi.org/10.1016/j. devcel.2019.10.019

Marín-Juez R, Marass M, Gauvrit S et al (2016) Fast revascularization of the injured area is essential to support zebrafish heart regeneration. Proc Natl Acad Sci 113:11237-11242. https://doi. org/10.1073/pnas.1605431113

Marro J, Pfefferli C, De Charles ASP et al (2016) Collagen XII contributes to epicardial and connective tissues in the zebrafish heart during ontogenesis and regeneration. PLoS ONE 11(10):e0165497. https://doi.org/10.1371/journal.pone.0165497

Masters M, Riley PR (2014) The epicardium signals the way towards heart regeneration. Stem Cell Res 13:683-692. https://doi. org/10.1016/j.scr.2014.04.007

Mirza R, DiPietro LA, Koh TJ (2009) Selective and specific macrophage ablation is detrimental to wound healing in mice. Am J Pathol 175:2454-2462. https://doi.org/10.2353/ajpat h. 2009.090248

Mollova M, Bersell K, Walsh S et al (2013) Cardiomyocyte proliferation contributes to heart growth in young humans. Proc Natl 
Acad Sci 110:1446-1451. https://doi.org/10.1073/pnas.12146 08110

Moore-Morris T, Guimarães-Camboa N, Banerjee I et al (2014) Resident fibroblast lineages mediate pressure overload-induced cardiac fibrosis. J Clin Invest 124:2921-2934. https://doi. org/10.1172/JCI74783

Morales RA, Allende ML (2019) Peripheral macrophages promote tissue regeneration in zebrafish by fine-tuning the inflammatory response. Front Immunol 10:253. https://doi.org/10.3389/fimmu .2019 .00253

Moyse BR, Richardson RJ (2020) A population of injury responsive lymphoid cells express mpeg 1.1 in the adult zebrafish heart. ImmunoHorizons 4(8):464-474. https://doi.org/10.4049/immun ohorizons.2000063

Müller P, Lemcke H, David R (2018) Stem cell therapy in heart diseases-cell types, mechanisms and improvement strategies. Cell Physiol Biochem 48:2607-2655. https://doi.org/10.1159/00049 2704

Münch J, Grivas D, González-Raja Á et al (2017) Notch signalling restricts inflammation and Serpine1 expression in the dynamic endocardium of the regenerating zebrafish heart. Development 144:1425-1440. https://doi.org/10.1242/dev.143362

Murry CE, Reinecke H, Pabon LM (2006) Regeneration gaps. J Am Coll Cardiol 47:1777-1785. https://doi.org/10.1016/j. jacc.2006.02.002

Nahrendorf M, Swirski FK, Aikawa E et al (2007) The healing myocardium sequentially mobilizes two monocyte subsets with divergent and complementary functions. J Exp Med 204:3037-3047. https://doi.org/10.1084/jem.20070885

Nauli SM, Jin X, AbouAlaiwi WA et al (2013) Non-motile primary cilia as fluid shear stress mechanosensors. Methods Enzymol 525:1-20

Nguyen-Chi M, Laplace-Builhé B, Travnickova J et al (2017) TNF signaling and macrophages govern fin regeneration in zebrafish larvae. Cell Death Dis 8:e2979-e2979. https://doi.org/10.1038/ cddis. 2017.374

Notari M, Ventura-Rubio A, Bedford-Guaus SJ et al (2018) The local microenvironment limits the regenerative potential of the mouse neonatal heart. Sci Adv 4(5):aao5553. https://doi.org/10.1126/ sciadv.aao5553

Ogryzko NV, Lewis A, Wilson HL et al (2019) Hif- $1 \alpha$-induced expression of Il-1 $\beta$ protects against mycobacterial infection in zebrafish. J Immunol 202:494-502. https://doi.org/10.4049/ jimmunol.1801139

Page DM, Wittamer V, Bertrand JY et al (2013) An evolutionarily conserved program of B-cell development and activation in zebrafish. Blood 122:e1-e11. https://doi.org/10.1182/blood -2012-12-471029

Patterson M, Barske L, Van Handel B et al (2017) Frequency of mononuclear diploid cardiomyocytes underlies natural variation in heart regeneration. Nat Genet 49:1346-1353. https:// doi.org/10.1038/ng.3929

Petrie TA, Strand NS, Yang C-T et al (2015) Macrophages modulate adult zebrafish tail fin regeneration. Development 142:406406. https://doi.org/10.1242/dev.120642

Pfefferli C, Jaźwińska A (2017) The careg element reveals a common regulation of regeneration in the zebrafish myocardium and fin. Nat Commun 8:15151. https://doi.org/10.1038/ncomms15151

Pinto AR, Ilinykh A, Ivey MJ et al (2016) Revisiting cardiac cellular composition. Circ Res 118:400-409. https://doi.org/10.1161/ CIRCRESAHA.115.307778

Porrello ER, Mahmoud AI, Simpson E et al (2011) Transient regenerative potential of the neonatal mouse heart. Science 331(6020):1078-1080. https://doi.org/10.1126/science.12007 08
Poss KD (2002) Heart regeneration in zebrafish. Science 298:21882190. https://doi.org/10.1126/science.1077857

Postlethwait JH (2000) Zebrafish comparative genomics and the origins of vertebrate chromosomes. Genome Res 10:1890-1902. https://doi.org/10.1101/gr.164800

Reiss K, Cheng W, Ferber A et al (1996) Overexpression of insulin-like growth factor- 1 in the heart is coupled with myocyte proliferation in transgenic mice. Proc Natl Acad Sci 93:86308635. https://doi.org/10.1073/pnas.93.16.8630

Robertson AL, Holmes GR, Bojarczuk AN et al (2014) A zebrafish compound screen reveals modulation of neutrophil reverse migration as an anti-inflammatory mechanism. Sci Transl Med 6(225):225-229. https://doi.org/10.1126/scitranslmed.3007672

Sacks CA, Jarcho JA, Curfman GD (2014) Paradigm shifts in heartfailure therapy - a timeline. N Engl J Med 371:989-991. https ://doi.org/10.1056/NEJMp1410241

Sánchez-Iranzo H, Galardi-Castilla M, Sanz-Morejón A et al (2018) Transient fibrosis resolves via fibroblast inactivation in the regenerating zebrafish heart. Proc Natl Acad Sci 115:41884193. https://doi.org/10.1073/pnas. 1716713115

Sanz-Morejón A, García-Redondo AB, Reuter H et al (2019) Wilms tumor $1 \mathrm{~b}$ expression defines a pro-regenerative macrophage subtype and is required for organ regeneration in the zebrafish. Cell Rep 28:1296-1306.e6. https://doi.org/10.1016/j.celre p.2019.06.091

Sanz-Morejón A, Mercader N (2020) Recent insights into zebrafish cardiac regeneration. Curr Opin Genet Dev 64:37-43. https:// doi.org/10.1016/j.gde.2020.05.020

Sattler S, Fairchild P, Watt FM et al (2017) The adaptive immune response to cardiac injury - the true roadblock to effective regenerative therapies? NPJ Regen Med 2:19. https://doi. org/10.1038/s41536-017-0022-3

Schnabel K, Wu CC, Kurth T, Weidinger G (2011) Regeneration of cryoinjury induced necrotic heart lesions in zebrafish is associated with epicardial activation and cardiomyocyte proliferation. PLoS ONE 6(4):e18503. https://doi.org/10.1371/journ al.pone. 0018503

Schneider L, Clement CA, Teilmann SC et al (2005) PDGFR $\alpha \alpha$ signaling is regulated through the primary cilium in fibroblasts. Curr Biol 15:1861-1866. https://doi.org/10.1016/j. cub.2005.09.012

Scott A, Ballesteros LS, Bradshaw M et al (2019) In vivo characterisation of endogenous cardiovascular extracellular vesicles in larval and adult zebrafish. BioRxiv. https://doi.org/10.1101/742692

Senyo SE, Steinhauser ML, Pizzimenti CL et al (2013) Mammalian heart renewal by pre-existing cardiomyocytes. Nature 493:433436. https://doi.org/10.1038/nature11682

Sertori R, Trengove M, Basheer F et al (2016) Genome editing in zebrafish: a practical overview. Brief Funct Genom 15:322-330. https://doi.org/10.1093/bfgp/elv051

Shiba Y, Gomibuchi T, Seto T et al (2016) Allogeneic transplantation of iPS cell-derived cardiomyocytes regenerates primate hearts. Nature 538:388-391. https://doi.org/10.1038/nature19815

Simões FC, Cahill TJ, Kenyon A et al (2020) Macrophages directly contribute collagen to scar formation during zebrafish heart regeneration and mouse heart repair. Nat Commun 11:600. https ://doi.org/10.1038/s41467-019-14263-2

Soonpaa MH, Kim KK, Pajak L et al (1996) Cardiomyocyte DNA synthesis and binucleation during murine development. Am J Physiol Circ Physiol 271:H2183-H2189. https://doi.org/10.1152/ ajpheart.1996.271.5.H2183

Stockdale WT, Lemieux ME, Killen AC et al (2018) Heart regeneration in the mexican cavefish. Cell Rep 25:1997-2007.e7. https://doi. org/10.1016/j.celrep.2018.10.072 
Tehzeeb J, Manzoor A, Ahmed MM (2019) Is Stem cell therapy an answer to heart failure: a literature search. Cureus 11(10):e5959. https://doi.org/10.7759/cureus.5959

Todorova D, Simoncini S, Lacroix R et al (2017) Extracellular vesicles in angiogenesis. Circ Res 120:1658-1673. https://doi. org/10.1161/CIRCRESAHA.117.309681

Vagnozzi RJ, Maillet M, Sargent MA et al (2020) An acute immune response underlies the benefit of cardiac stem cell therapy. Nature 577(7790):405-409. https://doi.org/10.1038/s41586-019-1802-2

Van Niel G, D'Angelo G, Raposo G (2018) Shedding light on the cell biology of extracellular vesicles. Nat Rev Mol Cell Biol 19(4):213-228. https://doi.org/10.1038/nrm.2017.125

Varda-Bloom N, Leor J, Ohad DG et al (2000) Cytotoxic T lymphocytes are activated following myocardial infarction and can recognize and kill healthy myocytes in vitro. J Mol Cell Cardiol 32:2141-2149. https://doi.org/10.1006/jmcc.2000.1261

Villalobos E, Criollo A, Schiattarella GG et al (2019) Fibroblast primary cilia are required for cardiac fibrosis. Circulation 139(20):2342-2357. https://doi.org/10.1161/CIRCULATIO NAHA.117.028752

Vivien CJ, Pichol-Thievend C, Sim CB et al (2019) Vegfc/d-dependent regulation of the lymphatic vasculature during cardiac regeneration is influenced by injury context. NPJ Regen Med 4:18. https ://doi.org/10.1038/s41536-019-0079-2

Wan F, Hu C, Ma J et al (2017) Characterization of $\gamma \delta$ T cells from zebrafish provides insights into their important role in adaptive humoral immunity. Front Immunol 7:675. https://doi. org/10.3389/fimmu.2016.00675

Wang J, Cao J, Dickson AL, Poss KD (2015) Epicardial regeneration is guided by cardiac outflow tract and Hedgehog signalling. Nature 522(7555):226-230. https://doi.org/10.1038/nature14325

Wang J, Karra R, Dickson AL, Poss KD (2013) Fibronectin is deposited by injury-activated epicardial cells and is necessary for zebrafish heart regeneration. Dev Biol 382:427-435. https://doi. org/10.1016/j.ydbio.2013.08.012

Wang J, Panáková D, Kikuchi K et al (2011) The regenerative capacity of zebrafish reverses cardiac failure caused by genetic cardiomyocyte depletion. Development 138:3421-3430. https://doi. org/10.1242/dev.068601

Wang X, Huang W, Liu G et al (2014) Cardiomyocytes mediate antiangiogenesis in type 2 diabetic rats through the exosomal transfer of miR-320 into endothelial cells. J Mol Cell Cardiol 74:139150. https://doi.org/10.1016/j.yjmcc.2014.05.001
Wang Y, Davidow L, Arvanites AC et al (2012) Glucocorticoid compounds modify smoothened localization and hedgehog pathway activity. Chem Biol 19(8):972-982. https://doi.org/10.1016/j. chembiol.2012.06.012

Wittamer V, Bertrand JY, Gutschow PW, Traver D (2011) Characterization of the mononuclear phagocyte system in zebrafish. Blood 117:7126-7135. https://doi.org/10.1182/blood-2010-11-321448

Xu S, Webb SE, Lau TCK, Cheng SH (2018) Matrix metalloproteinases (MMPs) mediate leukocyte recruitment during the inflammatory phase of zebrafish heart regeneration. Sci Rep 8:7199. https://doi.org/10.1038/s41598-018-25490-w

Xu S, Xie F, Tian L et al (2019) Prolonged neutrophil retention in the wound impairs zebrafish heart regeneration after cryoinjury. Fish Shellfish Immunol 94:447-454. https://doi.org/10.1016/j. fsi.2019.09.030

Yacoub M (2015) Cardiac donation after circulatory death: a time to reflect. Lancet 385:2554-2556. https://doi.org/10.1016/S0140 -6736(15)60683-3

Yang Z, Day Y-J, Toufektsian M-C et al (2006) Myocardial infarctsparing effect of adenosine $\mathrm{A} 2 \mathrm{~A}$ receptor activation is due to its action on CD4 + T LYMPHOCYTES. Circulation 114:20562064. https://doi.org/10.1161/CIRCULATIONAHA.106.649244

Ye L, D'Agostino G, Loo SJ et al (2018) Early regenerative capacity in the porcine heart. Circulation 138(24):2798-2808. https://doi. org/10.1161/CIRCULATIONAHA.117.031542

Ye L, Qiu L, Zhang H et al (2016) Cardiomyocytes in young infants with congenital heart disease: a three-month window of proliferation. Sci Rep 6:23188. https://doi.org/10.1038/srep23188

Yuan X, Cao J, He X et al (2016) Ciliary IFT80 balances canonical versus non-canonical hedgehog signalling for osteoblast differentiation. Nat Commun 7:11024. https://doi.org/10.1038/ncomm s11024

Zhou H, Wang B, Yang Y et al (2019) Exosomes in ischemic heart disease: novel carriers for bioinformation. Biomed Pharmacother 120:109451. https://doi.org/10.1016/j.biopha.2019.109451

Zhu W, Zhang E, Zhao M et al (2018) Regenerative potential of neonatal porcine hearts. Circulation 138(24):2809-2816. https://doi. org/10.1161/CIRCULATIONAHA.118.034886

Publisher's Note Springer Nature remains neutral with regard to jurisdictional claims in published maps and institutional affiliations. 